$44 \mid 2010$

De l'École de préparation des professeurs de français à l'étranger à l'UFR DFLE. Histoire d'une institution (1920-2008)

\title{
Note sur la création de l'École de préparation des professeurs de français à l'étranger
}

Daniel Coste

\section{QpenEdition \\ Journals}

Édition électronique

URL : https://journals.openedition.org/dhfles/2739

DOI : $10.4000 /$ dhfles.2739

ISSN : 2221-4038

Éditeur

Société Internationale pour l'Histoire du Français Langue Étrangère ou Seconde

Édition imprimée

Date de publication : 1 janvier 2010

Pagination : 29-39

ISSN : 0992-7654

Référence électronique

Daniel Coste, « Note sur la création de l'École de préparation des professeurs de français à l'étranger », Documents pour l'histoire du français langue étrangère ou seconde [En ligne], 44 | 2010, mis en ligne le 31 janvier 2014, consulté le 31 mars 2023. URL : http://journals.openedition.org/dhfles/2739 ; DOI : https://doi.org/10.4000/dhfles.2739

Ce document a été généré automatiquement le 31 mars 2023.

Tous droits réservés 


\title{
Note sur la création de l'École de préparation des professeurs de français à l'étranger
}

\author{
Daniel Coste
}

C'est en 1920 et à l'initiative de Ferdinand Brunot, alors doyen de la faculté des lettres de l'université de Paris, qu'est créée l'École de préparation des professeurs de français à l'étranger (EPPFE). Le choix de l'intitulé de l'institution donne lieu à des hésitations et débats dont les enjeux ne sont pas seulement terminologiques. La présente note vise à le montrer, tout en rappelant le modèle organisationnel et pédagogique prévu par les fondateurs ${ }^{1}$.

\section{1. École ou Institut?}

\section{1. École normale?}

2 En date du 18 février 1920, le vice-recteur de l'université de Paris adresse au ministre de l'Instruction publique et des Beaux-Arts un courrier relatif au projet de création d'une «École normale des professeurs de français à l'étranger ". L'initiative vient du doyen de la faculté des lettres, Ferdinand Brunot. Le vice-recteur ne fait pas preuve d'un grand enthousiasme et manifeste à tout le moins un souci de ne pas trop engager la Sorbonne dans l'entreprise :

L'école en question ne saurait, sans de graves difficultés, être rattachée directement

à l'Université de Paris; mais l'Université lui accorderait bien volontiers son patronage. [Au demeurant], il serait plus expédient de charger la Société des Amis de l'Université de l'organisation administrative de cette école.

Le projet de Ferdinand Brunot, tel qu'il apparaît dans le courrier du vice-recteur est déjà détaillé pour cette école qui serait ouverte aux hommes et aux femmes, français ou étrangers, âgés de 18 ans au moins et pour lesquels aucune condition préalable de niveau de diplôme n'est formellement posée. Le comité de direction prévu se composerait de Ferdinand Brunot lui-même, de Gustave Lanson, alors directeur de 
l'École normale supérieure de la rue d'Ulm, d'Edmond Huguet, professeur à la Sorbonne et de Mario Roques, professeur à l'École des Langues orientales².

\section{2. Institut?}

4 Le $1^{\text {er }}$ mai 1920 , une commission préparatoire se réunit, accueillie à l'ENS par Lanson. Elle examine le positionnement de l'école projetée, estime qu'elle n'entrera en concurrence ni avec l'Alliance française (que Brunot connaît bien ${ }^{3}$ ), ni avec les Cours de civilisation française de la Sorbonne. Contrairement à l'avis qu'avait exprimé au ministre le vice-recteur, la commission, qui rebaptise «Institut d'enseignement du français à l'étranger " ce qui, quelques semaines plus tôt, s'appelait encore "École normale ", considère que ledit institut devrait être rattaché à l'université. Le petit groupe examine aussi le montage financier de l'opération. Le doyen Brunot se dit assuré d'une contribution de la Propagande ${ }^{4}$ de l'ordre de 10000 à 100000 francs et suggère que l'université s'engage de son côté pour une durée de deux à trois ans, sorte de période d'essai pour la viabilité du projet, l'institut étant appelé à percevoir par ailleurs des frais d'études. Les bénéfices éventuels reviendraient à l'université, à charge pour elle de procéder à des reversements à l'Institut.

\section{3. École spéciale ou institut?}

5 Cette réunion du $1^{\text {er }}$ mai était à l'évidence destinée à préparer en groupe restreint la réunion du Conseil de l'université, qui, tenue le 3 mai, examine à nouveau le projet. Brunot propose un troisième intitulé: "École spéciale des professeurs de français à l'étranger ", placée à l'intérieur de l'université sous la direction de la faculté des lettres. Lanson diverge: il rappelle son opposition à "École normale ", trouve ambiguë la désignation "École spéciale » et préfère s'en tenir à "Institut », comme lors de la réunion préparatoire. Mais, pour Brunot, cela ne convient plus, car l'appellation « institut » ne peut s'appliquer qu'à un lieu de recherche. À l'issue de ces échanges, on passe aux voix et le doyen des lettres est mis en minorité. Le Conseil de l'université retient «Institut de préparation des professeurs de français à l'étranger, sous la direction de la faculté des lettres».

\section{4. Dispositions organisationnelles}

6 Le même Conseil accepte la proposition selon laquelle le comité de direction de la future instance sera composé de :

7 - quatre professeurs de la faculté des lettres

8 - un professeur de la faculté de droit

9 - un professeur de l'École normale supérieure

10 - le directeur de l'Institut

11 - le secrétaire général de l'Institut

12 - un membre de l'enseignement secondaire

13 - un membre de l'enseignement primaire

14 - un membre de la Chambre de commerce. 
Le Conseil s'interroge sur le point de savoir s'il y a lieu d'ajouter à ce comité de direction un représentant de l'Alliance française, mais cette suggestion est écartée, au motif qu'il faudrait alors aussi un représentant de la Mission laïque ${ }^{5}$.

Brunot propose le nom d'Edmond Huguet pour les fonctions de directeur et celui de Léopold Sudre, professeur au lycée Louis-le-Grand pour exercer celles de secrétaire général ${ }^{6}$.

Le programme pédagogique est plus qu'esquissé. Il comprend notamment, à l'examen final, «l'exposé dogmatique d'une question de grammaire » (dont Brunot souligne que seule alors l'agrégation de jeunes filles le prévoit). On n'exclut pas que les enseignements du programme puissent être complétés par certains de ceux des Cours de civilisation de la Sorbonne ${ }^{7}$ ou de l'Alliance française. Sera délivré un «Diplôme de l'Université de Paris pour l'enseignement du français à l'étranger ».

8 Le lancement de l'institut projeté bénéficiera d'une subvention exceptionnelle de 100000 francs, attribuée soit par le ministère, soit par la Propagande. L'université doit s'engager à " parfaire le déficit ", si le cas se présente, car " un intérêt national est en jeu ».

\section{Ce sera une école !}

Au-delà de ce parcours interne à l'université, il revient au ministère de l'Instruction publique et des Beaux-Arts de prendre l'arrêté de création. C'est ce qui est fait en date du 27 octobre 1920. Et, que Brunot soit intervenu ou non en ce sens, c'est bien d'une école et non d'un institut qu'il s'agira au bout de ce compte. L'École de préparation des professeurs de français à l'étranger est instituée. "Elle se propose de donner à tous les professeurs de français à l'étranger des disciplines, une méthode ».

La vaste ambition ainsi déclarée se décline selon une dizaine de sections dans le plan d'études, qui se compose comme suit:

- révision des connaissances relatives à l'histoire, géographie, civilisation de la France

- vues sommaires sur les divers pays où les professeurs doivent être envoyés

- littérature française

- phonétique du français

- lecture à haute voix

- vocabulaire français

- grammaire du français

- orthographe du français

- composition française

- explication de textes.

L'examen de fin d'études prévoit, pour l'écrit (éliminatoire) :

- un commentaire grammatical de passages d'auteurs des XVI ${ }^{\mathrm{e}}, \mathrm{XVII}^{\mathrm{e}}$ ou XVIII ${ }^{\mathrm{e}}$ siècles

- une composition française sur un sujet relatif à un des auteurs du programme.

Et pour l'oral : 
35 - la lecture à haute voix et l'explication littérale et littéraire d'un texte choisi par le jury parmi les auteurs du $\mathrm{XIX}^{\mathrm{e}}$ ou du $\mathrm{xx}^{\mathrm{e}}$ siècle

36 - l'exposé dogmatique d'une question de grammaire

37 - un exposé relatif à l'histoire, la géographie ou la phonétique.

38 L'arrêté dispose aussi que les élèves français de l'École pourront être admis s'ils sont titulaires du baccalauréat ou du brevet supérieur ou de diplômes de fin d'études des lycées et collèges de jeunes filles.

39 Le cadre est en place. Il ne reste plus à l'EPPFE qu'à faire ses preuves.

\section{Quelques commentaires}

40 - Le doyen Ferdinand Brunot a donc réussi à mener à terme le projet, caressé dès longtemps, de créer un lieu universitaire de formation d'enseignants de français pour l'étranger. La Grande Guerre n'avait pas permis que l'affaire se fasse plus tôt, mais Brunot y songeait sans doute depuis qu'il intervenait dans les cours d'été de l'Alliance française s'adressant en particulier à des enseignants ou futurs enseignants étrangers de français. La nouvelle école accueille aussi des Français et peut délivrer, n'en déplaise au vice-recteur, un diplôme de l'université de Paris, ce qui n'était pas possible dans un cadre associatif. L'ancrage universitaire distingue l'École non seulement des associations telles que l'Alliance ou la Mission laïque, mais aussi des Cours de civilisation française de la Sorbonne, de création légèrement antérieure mais de statut d'association.

41 - Une telle création n'est pas tout à fait la première du genre. En Suisse romande, l'université de Genève en 1891 , celles de Lausanne et de Neuchâtel ${ }^{8}$ ont mis en place des Séminaires de français moderne, qui, en enseignements annuels ou en cours d'été, assurent des formations certifiantes intéressant notamment les enseignants allemands de français. En France, en liaison avec l'université de Grenoble, un "Comité de patronage " ouvre des cours à la fin des années 1890, qui auront un succès certain ; d'autant que Théodore Rosset développe parallèlement dans l'université et regroupe en un institut des travaux et recherches en phonétique qui intéressent aussi l'enseignement (Gruca 1997; Galazzi 1997). Mais, pas plus que dans les universités suisses, il ne s'agit d'une école de préparation d'enseignants de français à l'étranger délivrant comme telle un diplôme d'université.

42 - Les pères fondateurs, Brunot, Lanson, Huguet et Roques, ont pour traits communs peu surprenants pour l'époque - d'être tous agrégés (de lettres pour Lanson, de grammaire pour les trois autres) et d'anciens élèves de l'École normale supérieure de la rue d'Ulm (voir Chevalier avec Encrevé 2006). Ils appartiennent à des générations universitaires où l'histoire littéraire (Lanson), la philologie, l'histoire de la langue et la grammaire historique (Brunot, Huguet) marquent les études de français. Mario Roques, quant à lui, est alors surtout philologue romaniste, attaché à l'ancien français ${ }^{9}$. Ce sont des modernistes, bien que formés aux études classiques et l'on sait quel rôle Ferdinand Brunot tient aussi pour ce qui est de l'enseignement du français langue maternelle (Chevalier avec Encrevé 2006 ; Besse 1995).

43 - L'histoire ultérieure de l'EPPFE restera marquée, quant à sa direction, par les spécialistes de français moderne, d'histoire de la langue ou de phonétique (Saulnier, 
seiziémiste comme Huguet, Fouché, phonéticien), jusqu'à Bernard Quemada, à partir de qui s'ouvre pour l'institution une autre reconnaissance dans la structure universitaire.

44 - On pourrait être surpris par l'absence dans le plan d'études de ce qui serait désigné aujourd'hui comme formation didactique ou méthodologique. Mais c'est que ces dimensions se ramènent en fait à des propositions de démarches pédagogiques et d'exercices à l'intérieur de chacune des branches du programmes (vocabulaire, lecture, orthographe, etc.). L'examen final semble centrer l'appréciation sur la maîtrise de ces genres scolaires "à la française », qui marqueront durablement l'enseignement du français à l'étranger : composition française, leçon de grammaire, explication de texte littéraire.

45 - Pour Ferdinand Brunot, l'institution «école normale ", autre que supérieure, a son plein sens ; il s'agit, dans cette Troisième République patriotique et militante de former des enseignants qui seront des propagateurs du français à l'étranger, tout comme le sont en France les maîtres sortis des écoles normales d'instituteurs et d'institutrices ${ }^{10}$. Directeur de la prestigieuse École normale supérieure de la rue d'Ulm, Lanson, partisan pour le projet d'un intitulé «institut » est peut-être soucieux d'éviter les confusions que pourrait entretenir, quelles que soient les différences de statut et de niveau, l'existence d'une « école normale » à l'intérieur de la non moins prestigieuse Sorbonne.

46 - Le doyen des lettres Brunot, rappelant que la désignation institut ne saurait être retenue, implique aussi que l'École n'aura pas vocation à la recherche. Choix évidemment légitime à l'époque, compte tenu de la fonction majeure de l'établissement et du fait que le "français à l'étranger " n'est pas considéré vraiment dans une perspective différente de celle retenue pour le français en France (Coste 2003). L'avènement $d u$ " français langue étrangère » attendra une quarantaine d'années et la création du CREDIF (Centre de recherche et d'étude pour la diffusion du français), du Centre de linguistique appliquée de Besançon, du BELC (Bureau pour l'enseignement de la langue et de la civilisation françaises), de la revue Le Français dans le monde. De façon significative, c'est en 1963, juste après ces diverses créations, que l'ancienne École de préparation des professeurs de français à l'étranger, déjà " promue » en 1945 «École supérieure de préparation et de perfectionnement des professeurs de français à l'étranger ", accède enfin au statut d'Institut et devient l'IPFE, l'Institut des professeurs de français à l'étranger.

47 - Ce jeu entre «Institut » et «École » (normale ou non...) et les fluctuations initiales d'appellation ne sont pas seulement de circonstance, de personne ou d'époque. Ce qui est ainsi manifesté, dès l'origine, c'est la difficulté de positionnement de telles instances de formation à l'intérieur des structures universitaires. Séminaires, écoles, centres de français pour étrangers, centres de langues rencontrent jusqu'à aujourd'hui des difficultés et des ambiguïtés de reconnaissance et d'insertion dans l'Alma Mater et les débats ou crises n'ont pas manqué au fil des années autour de ces enjeux (Coste 1998).

48 - Reste que, s'agissant de donner corps et visibilité universitaire à des initiatives nouvelles n'entrant pas dans l'ordre strict de l'organisation des facultés et de leur découpage académique, l'instance « institut » est particulièrement productive pour la Sorbonne du début du siècle dernier. Les « Archives de la parole », créées par Ferdinand Brunot deviendront, grâce au même, en 1911, l'Institut de phonétique de Paris (qui concerne alors surtout la phonétique historique) ${ }^{11}$. Dans la période même où naît l'EPPFE, un décret du 31 juillet 1920 réglemente l'organisation des instituts de 
l'université. Et c'est à ce décret que peut se référer un autre décret (et non pas un arrêté comme pour l'EPPFE) qui, en date du 17 décembre 1921, porte création d'un Institut de linguistique. C'est un autre quatuor qui porte cet institut sur les fonts baptismaux, composé de Joseph Vendryes, Antoine Meillet, Oscar Bloch et Paul Boyer ${ }^{12}$. Il vise à regrouper ce qui se fait en matière de recherche linguistique et ses statuts prévoient des contacts avec d'autres instituts: Institut de phonétique, Institut de psychologie, Institut de géographie. De l'EPPFE, il n'est pas question. Brunot et Meillet se trouvent bien, comme le souligne Jean-Claude Chevalier (2006: 15-20) en complémentarité ${ }^{13}$.

\section{BIBLIOGRAPHIE}

BESSE, Henri (1995). « Ferdinand Brunot, méthodologue de l'enseignement de la langue française », Histoire Épistémologie Langage, XVII-1, 41-74.

BRUÉZIÈRE, Maurice (1983). L'Alliance française. Paris : Hachette.

CHEVALIER, Jean-Claude (1997). «L'introduction progressive de la didactique des langues dans l'Institution universitaire ", Documents pour l'histoire du français langue étrangère ou seconde, 20, (L'apport des centres de français langue étrangère à la didactique des langues), 9-21.

- (2001). «Diffusion du français en Europe de l'Est : 1920-1939 », in Kok Escalle, M.-C. et Melka, F. (réd.), Changements politiques et statut des langues. Histoire et épistémologie 1780-1945. AmsterdamAtlanta : Rodopi.

CHEVALIER, J.-Cl. et ENCREVÉ, Pierre (éds) (1984). « Vers une histoire sociale de la linguistique », Langue française, 63.

CHEVALIER, J.-Cl. avec ENCREVÉ, Pierre (2006). Combats pour la linguistique, de Martinet à Kristeva. Essai de dramaturgie épistémologique. Lyon : ENS Éditions.

CHISS, Jean-Louis (1995). « À partir de Bally et Brunot : la langue française, les savants et les pédagogues ", Histoire Épistémologie Langage, XVII-1, 19-40.

COSTE, Daniel (1991). « La fondation du séminaire de français moderne de Genève en 1891 : moment des relations entre université et enseignement des langues », Bulletin CILA, 54, 39-50.

- (1998). «La gravité des centres : attractions et pesanteurs », in F. Grossmann (coord.), Pratiques langagières et didactiques de l'écrit. Hommage à Michel Dabène. Université Stendhal-Grenoble III : IVEL-LIDILEM, 61-72.

- (2003). «Entre mythe linguistique et politique des langues ", Triangle 19, Les langues maternelles dans l'enseignement des langues étrangères. Lyon : ENS Éditions, 15-30.

GALAZZI, Enrica (1997). « Théodore Rosset et l'établissement de la phonétique expérimentale », Documents pour l'histoire du français langue étrangère ou seconde, 20 (L'apport des centres de français langue étrangère à la didactique des langues), 37-53.

GRUCA, Isabelle (1997). « Les enseignements organisés par le Comité de Patronage des Étudiants Étrangers de 1896 à 1975 : entre modernisme et tradition ", Documents pour l'histoire du français 
langue étrangère ou seconde, 20 (L'apport des centres de français langue étrangère à la didactique des langues), 86-99.

SAUGY, Catherine (1987). L'École de français moderne de l'Université de Lausanne, 1892-1987.

Université de Lausanne.

- (1997). «L'occasion fait le larron : aux origines du français langue étrangère à l'Université de

Lausanne ", Documents pour l'histoire du français langue étrangère ou seconde, 20 (L'apport des

centres de français langue étrangère à la didactique des langues), 117-126.

SWIGGERS, Pierre (2004). « Aux côtés d'Arsène Darmesteter : Léopold Sudre ", Revue des Langues Romanes, 108-[2], 483-503.

TERRIER, Philippe (1997). « 100 ans d'enseignement du français langue étrangère à l'Université de Neuchâtel (1892-1992) ", Documents pour l'histoire du français langue étrangère ou seconde, 20

(L'apport des centres de français langue étrangère à la didactique des langues), 127-140.

THÉVENIN, André (2002). La Mission laïque française à travers son histoire. Paris : Mission laïque française.

\section{NOTES}

1. Je tiens à remercier les collègues du Service des archives du Rectorat de Paris qui m'ont donné accès aux dossiers concernant l'EPPFE.

2. Ce dernier, né en 1875, est le plus jeune. Brunot, Lanson et Huguet sont respectivement nés en 1860, 1857, 1863. Brunot est doyen de la faculté des lettres depuis 1919.

3. Brunot a été très actif à l'Alliance, où il a assuré, après 1895 , la direction des cours d'été.

4. «La Propagande» désigne sans doute ici les organismes de propagande coloniale et notamment l'Agence générale des colonies, créée en 1919, à partir d'instances similaires dans la mouvance du ministère des Colonies. Il faut rappeler à ce propos que les statuts originaux de l'Alliance française et de la Mission laïque française prévoient aussi l'intervention dans le cadre colonial (Bruézière 1983, Thévenin 2002). L'enseignement aux étrangers et l'enseignement colonial sont complémentaires. C'est toutefois le ministère de l'Instruction publique et des Beaux-Arts, non la Propagande, qui accordera la subvention de départ de la nouvelle institution voulue par Brunot.

5. L'Alliance française a été fondée en 1883, la Mission laïque française en 1902.

6. Léopold Sudre (1855-1932), docteur ès-lettres et spécialiste du Roman de Renart, est l'auteur d'une Chrestomathie du Moyen Âge et a été un proche collaborateur de Darmesteter (cf. Swiggers 2004).

7. Gérés par la Société des Amis des Universités de Paris, qui fut créée en 1899 à des fins sociales et humanitaires, les Cours de civilisation française de la Sorbonne ont été fondés en 1919, peu avant l'École de préparation des professeurs. On comprend que le vice-recteur ait proposé d'adopter le même type de rattachement pour le projet de Brunot que pour ces Cours.

8. A propos de ces séminaires, voir Coste $(1991)$, Saugy $(1987,1997)$ et Terrier (1997). À noter que Ferdinand de Saussure est associé au suivi du Séminaire de français moderne de Genève, où Charles Bally assure des cours.

9. Brunot, Huguet et Roques ont tous trois, dès avant 1914, fait partie d'un comité assistant la direction de l'École pratique de l'Alliance française, sorte de banc d'essai hors université de l'EPPFE

10. Cette référence à l'institution école normale existe aussi du côté des associations militantes. Ce n'est pas tout à fait un hasard si la Mission laïque française, fondée et animée, entre autres, 
par des anciens de l'École normale supérieure de Saint-Cloud, met en place dès sa fondation une « école normale d'enseignement colonial».

11. L'Institut de phonétique de Paris, n'est pas le premier du genre. Celui de Grenoble, dû à Rosset et plus orienté vers la phonétique expérimentale, date de 1904.

12. Tous linguistes indo-européanistes de formation. Meillet, Vendryes et Bloch sont agrégés de grammaire. Paul Boyer, slavisant, est directeur de l'École des Langues orientales.

13. «... différents dans la mesure où ils sont complémentaires : Brunot est un enseignant qui forme des enseignants pour les écoles, les lycées et les collèges; sa discipline est inséparable de l'enseignement $d u$ français, de son histoire et de l'étude des textes. Il est philologue au sens propre [...]. Meillet est linguiste comme on l'est en France à l'époque : il privilégie le domaine indo-européen; il étudie l'arménien aussi bien que les langues slaves; ce jeu acrobatique entre des langues exotiques exige un attirail théorique au moins minimal» (Chevalier 2006 : 15-16).

\section{RÉSUMÉS}

En 1920, à l'initiative de Ferdinand Brunot, alors doyen de la faculté des lettres de l'université de Paris, est créée l'École de préparation des professeurs de français à l'étranger (EPPFE). Le choix de l'intitulé de l'institution a donné lieu à des hésitations et débats dont les enjeux ne sont pas seulement terminologiques. La présente note vise à le montrer, tout en rappelant le modèle organisationnel et pédagogique prévu par les fondateurs.

L'École de préparation des professeurs de français à l'étranger* (EPPFE) originates in 1920 from a project initiated by Ferdinand Brunot, then Dean of the faculté des lettres de l'université de Paris. Deciding on a name and statute for the new institution gave rise to some fluctuations and discussions and the issues at stake were not just of a terminological nature. The present notice illustrates this point and recalls the organizational and pedagogical model chosen by the founders of the school.

* «Training school for teachers of French abroad».

\section{INDEX}

Mots-clés : Brunot, École de préparation des professeurs de français à l'étranger, EPPFE, institutions

Keywords : Brunot, EPPFE, institutions, Training school for teachers of French abroad

\section{AUTEUR}

DANIEL COSTE

École normale supérieure de Lyon 